\title{
Foix-Chavany-Marie syndrome due to type E TDP43 pathology
}

\author{
Authors: CN Clark ${ }^{1, *}$ PhD MRCP, A Quaegebeur ${ }^{2, *}$ MD PhD, N Nirmalananthan ${ }^{1}$ PhD FRCP, \\ Andrew D MacKinnon ${ }^{3}$ MD FRCR, Tamas Revesz ${ }^{4}$ MD PhD FRCPath, JL Holton ${ }^{2}$ PhD \\ FRCPath, LR Bridges ${ }^{5}$ MD FRCPath, AC Pereira ${ }^{1}$ MD FRCP \\ ${ }^{1}$ Neurology department, Atkinson Morley Regional Neuroscience Centre, St \\ George's University Hospitals NHS Foundation Trust, London, UK \\ ${ }^{2}$ Division of Neuropathology, National Hospital for Neurology and \\ Neurosurgery, University College London Hospitals NHS Foundation Trust, \\ London, UK \\ ${ }^{3}$ Department of Neuroradiology, Atkinson Morley Regional Neuroscience \\ Centre, St George's University Hospitals NHS Foundation Trust, London, UK. \\ ${ }^{4}$ Queen Square Brain Bank for Neurological Disorders and Department of \\ Neurodegenerative Disease, UCL, Queen Square Institute of Neurology, \\ London, UK. \\ ${ }^{5}$ St George's University Hospitals NHS Foundation Trust, Department of \\ Cellular Pathology, St George's Hospital, Blackshaw Road, London SW17 0QT \\ * joint first authors as contributed equally to final manuscript
}

Corresponding author: Anthony Pereira

\author{
St George's Hospital \\ Blackshaw Road \\ London SW17 0QT \\ Phone: 02087254322 \\ Fax: 02087254700 \\ anthony.pereira@stgeorges.nhs.uk
}

\section{Study funding}

CN Clark reports no disclosures

A Quaegebeur reports no disclosures

$\mathrm{N}$ Nirmalananthan reports no disclosures

AD MacKinnon reports no disclosures

T Revesz is supported by the Karin \& Sten Morstedt CBD Solutions AB and the National Institute for Health Research (NIHR) Queen Square Biomedical Reseach Unit in Dementia based at University College London Hospitals (UCLH), University College London

$\mathrm{JL}$ Holton is supported by the Multiple System Atrophy Trust and Karin \& Sten Mortstedt CBD Solutions.

LR Bridges reports no disclosures

AC Pereira reports no disclosures

This research was supported by the National Institute for Health Research University College London Hospitals Biomedical Research Centre. The Editors of Neuropathology and Applied Neurobiology are committed to peer-review integrity and upholding the highest standards of review. As such, this article was peer-reviewed by independent, anonymous expert referees, and the authors (including $\mathrm{JH}$ ) had no role in either the editorial decision or the handling of the paper. 
Character count for title: 57

Abstract: N/A

Word count main text: 1067

Figures: 1

References: 10

\section{Author Contributions}

CN Clark: Drafting manuscript and reviewing patient notes.

A Quaegebeur: Drafting manuscript and neuropathological diagnosis

N Nirmalananthan: Clinical management and diagnosis of patient in life, manuscript review

AD MacKinnon: Selection and review of MR images and editing of legend, manuscript Review

T Revesz: neuropathological diagnosis and manuscript review

JL Holton: neuropathological diagnosis and manuscript review

LR Bridges: neuropathological diagnosis and manuscript review

AC Pereira: Clinical management, critical revision of text and images for intellectual comment.

Search terms: Foix-Chavany-Marie, opercular syndrome, primary progressive aphasia, PPA, FTLD, frontotemporal lobar degeneration, TDP43 pathology, FTLD-TDP type E, granulofilamentous neuronal inclusions, GFNI 
We present a 78-year-old lady with a clinical and radiological diagnosis of anterior opercular syndrome or Foix-Chavany-Marie syndrome (FCMS) and an autopsy diagnosis of type E TDP43 pathology. The neurodegenerative form of FCMS presents with articulation difficulty, dysprosody, orofacial and speech apraxia and can mimic but is distinct from primary progressive aphasia (PPA) (1, 2). It was suggested that the progressive form of FCMS should be included with frontotemporal lobar degenerations (FTLD) (3). However, its neuropathological substrates are poorly understood. This case reveals a rare pattern of TDP43 pathology as neurodegenerative cause of FCMS.

The patient presented with falls and a rapidly progressive dysarthria on a background of hypertension. Within 4 months she was fed via nasogastric tube. She became rapidly cognitively impaired (not formally quantified owing to unintelligibility of speech). There was minimal voluntary facial movement (closing eyes/smiling to command) with preserved involuntary movements (blink to threat/ spontaneous laughter). Palatal elevation was normal with no tongue wasting or fibrillation. There was mild wasting and weakness of her hands but no significant wasting or fasciculations elsewhere. Power generally was good. She walked slowly, but unaided. Reflexes (including facial, jaw and trapezius) were normal. MR brain imaging showed widening of the Sylvian fissures and frontoparietal sulci, with right-sided predominance (Figure 1). A tentative diagnosis of motor neuron disease (MND) was initially entertained but without EMG support, the clinico-radiological diagnosis was of anterior opercular syndrome secondary to neurodegeneration.

No other specific radiological abnormalities were identified. CT thorax, abdomen and pelvis showed no malignancy. Repeat EMG/NCS one month later demonstrated mild corticospinal tract dysfunction with small potentials from hands and feet on transcranial magnetic stimulation. EMG showed mild neurogenic changes in the limb muscles and tongue. Therefore, despite evidence of upper and lower motor neurone dysfunction, the absence of evidence of denervation and reinnervation in the muscles failed to support the diagnosis of MND neurophysiologically, despite a high clinical index of suspicion. Videofluoroscopy showed abnormal contraction of the posterior pharyngeal wall.

A radiologically guided gastrostomy was inserted. A trial of steroids did not demonstrate improvement. She became increasingly weak (4/5 upper limbs and 3/5 lower limbs on MRC scale) with hyperreflexia. Within 6 months of presentation, she developed chronic type 2 respiratory failure and was palliated.

At autopsy, the brain weighed 1318g and showed atrophy of the inferior frontal and superior temporal gyri. Histologically there was superficial spongiosis and mild neuronal loss in the frontal and temporal lobes in keeping with frontotemporal lobar degeneration (FTLD). The other lobes, medial temporal lobe structures, deep grey nuclei and hindbrain showed preserved cytoarchitecture. Immunostaining for phosphorylated TDP43 showed finely granular neuronal cytoplasmic inclusions ( $\mathrm{NCls}$ ) with fine grain-like inclusions and short threads in the neuropil of all cortical layers of the temporal and frontal cortices with emphasis on the superficial layers. Compact $\mathrm{NCls}$ were rare and neuronal nuclear inclusions were not identified. Grey and white matter showed scattered glial cytoplasmic inclusions (figure 1). This pattern did not conform to the well described subtypes of TDP43 pathology (type A-D) (4) but was similar to the morphology of inclusions observed in the recently proposed FTLD-TDP type E (5). TDP43 pathology was more severe in the affected cortical regions of the right cerebral hemisphere, similar to the asymmetry found on MRI (table 1). TDP43 pathology in the hippocampus was sparse. Rare NCls were observed in the hypoglossal nuclei 
and increased microglial activation was noted in the pyramids indicative of corticospinal tract degeneration. In addition to TDP43 pathology there was evidence of low level of Alzheimer's disease pathological change (6) with amyloid-beta pathology corresponding to Thal phase 3 and neurofibrillary tangle and thread pathology not exceeding Braak\&Braak stage I. Vascular comorbidity was limited to presence of mild hyaline arteriosclerosis affecting the deep grey nuclei and both cerebral and cerebellar white matter. Overall the most significant neuropathological findings were those of FTLD due to TDP43 proteinopathy, Type E.

The neuropathological study of FCMS is limited to a handful of reports (7-10) all showing focal cortical degenerative pathology in keeping with FTLD. A single study described a clinical presentation of opercular syndrome in a patient carrying a P332S MAPT mutation (9) and showing tauopathy with Pick-like bodies on autopsy. A further study described the pathology in a patient with FCMS as TDP43-positive NCls in frontal and temporal lobes along with involvement of motor cortex and hypoglossal nucleus (8) in keeping with FTLD-TDP with MND. The pattern of TDP43 pathology described most likely corresponds to type A. Others found degeneration in keeping with FTLD with tau negative, ubiquitin-positive neuronal cytoplasmic inclusions, diagnosed as FTLD-U (10). TDP43 staining was not performed, but one may speculate that this might have been a case of TDP43 proteinopathy. Finally, two cases were described with focal frontal cortical atrophy (7) with Immunostaining for tau and ubiquitin described as "insignificant" in one case and showing "a certain number" of neuronal inclusions in the other. Whilst both cases seem consistent with FTLD the precise pathologies are unclear in today's terms.

The morphology and distribution of the TDP43 inclusions in this case is unusual, resembling a recently proposed subtype of TDP43 pathology, FTLD-TDP type E (5). This study described 7 cases of FTLD-TDP showing a distinct pattern of TDP43 pathology not corresponding to any of the existing TDP43 pathology subtypes, comprising granulofilamentous inclusions, abundant grains and oligodendroglial inclusions. All cases presented clinically with behavioural variant of FTD with a single case showing additional motor neuron disease. Whilst in the latter patient a C9orf72 mutation was detected, no other known mutations were identified. In our case genetic testing was not available, however, the absence of p62-positive, TDP43-negative inclusions in hippocampus and cerebellum argued against a C9orf72 repeat expansion mutation. Of interest, the short disease duration in our case (6 months) is in line with the rapidly progressive disease course characterising the previously described TDP43 type E cases (5).

To summarise, a 78-year-old lady with rapidly progressive pseudobulbar dysarthria with automaticvoluntary dissociation and cognitive impairment together with radiological evidence of an anterior opercular syndrome was found to have a rare pattern of TDP43 pathology corresponding to the newly proposed category of FTLD-TDP type E. Not only do our findings expand the clinical spectrum encountered in this distinct type of TDP43 pathology to anterior opercular syndrome, but also we describe one of the very few pathologically proven cases of neurodegenerative FCMS highlighting that TDP43 pathology should be included in the differential diagnosis. 

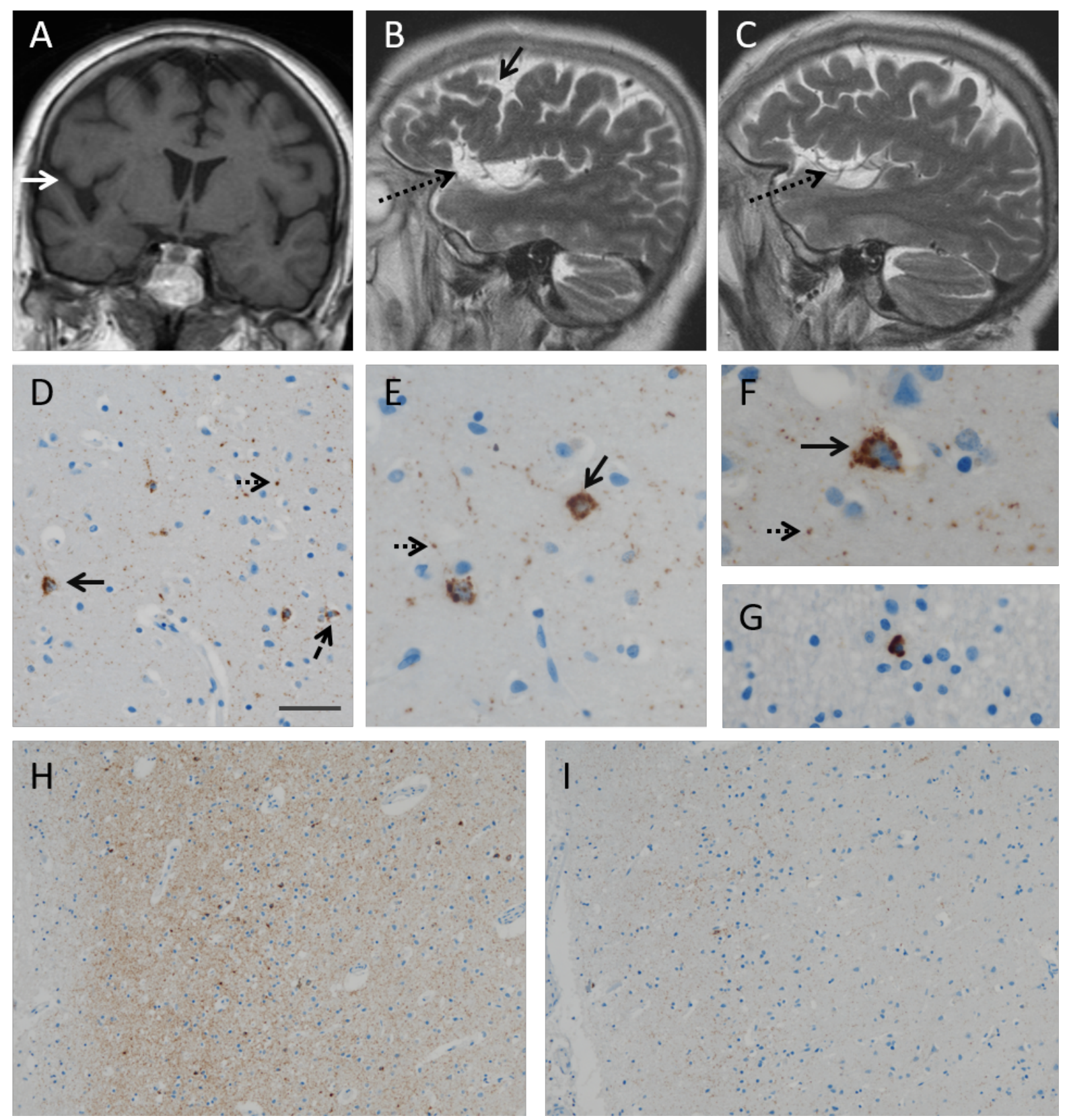

Figure 1: Radiological and histopathological features. MRI brain images (A-C). A: Coronal T1 image showing right predominant Sylvian fissure (white arrow) widening secondary to atrophy of the inferior frontal and superior temporal gyri; B: Sagittal T2 image showing widening of right precentral sulcus (black arrow) and atrophy of right anterior frontal operculum (dotted arrow); C: Sagittal T2 image showing less pronounced left anterior frontal opercular atrophy (dotted arrow). Immunohistochemical staining for phosphorylated TDP43 (D-I). D-F: Finely granular neuronal cytoplasmic inclusions (arrow) on a background of short threads and fine grain-like inclusions (dotted arrow) in the neocortex of the right superior temporal gyrus; occasional glial cytoplasmic inclusions (dashed arrow) are also present. G: Glial cytoplasmic inclusion in white matter of right superior temporal gyrus. H-I: TDP43 pathology in right $(\mathrm{H})$ and left $(\mathrm{I})$ inferior frontal gyrus highlighting left-right asymmetry in TDP43 pathology. Orientation in panel $\mathrm{H}$ and I demonstrate superficial cortex on the left and deep cortex on the right aspect. Bar in (D) represents $120 \mu \mathrm{m}$ in $(\mathrm{H}$,

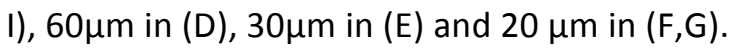


Table 1: Severity of TDP43 pathology across different brain regions

\begin{tabular}{lll} 
Brain region & Right & Left \\
\hline Superior frontal gyrus & +++ & ++ \\
Inferior frontal gyrus & +++ & ++ \\
Insula & ++ & + \\
Superior temporal gyrus & ++ & + \\
Inferior temporal gyrus & +++ & + \\
Occipital & - & + \\
Hippocampus & + & \\
&
\end{tabular}

Acknowledgements: JLH is supported by the Multiple System Atrophy Trust and Karin \& Sten Mortstedt CBD Solutions. TR is supported by the Karin \& Sten Morstedt CBD Solutions AB and the National Institute for Health Research (NIHR) Queen Square Biomedical Reseach Unit in Dementia based at University College London Hospitals (UCLH), University College London. This research was supported by the National Institute for Health Research University College London Hospitals Biomedical Research Centre. 


\section{References}

1. Uttner I, Brettschneider J, Unrath A, Riecker A. Slowly progressive Foix-Chavany-Marie syndrome as a precursor of a primary progressive aphasia. Journal of clinical neuroscience : official journal of the Neurosurgical Society of Australasia. 2012;19(5):765-7.

2. Weller M, Poremba M, Dichgans J. Opercular syndrome without opercular lesions: FoixChavany-Marie syndrome in progressive supranuclear motor system degeneration. European archives of psychiatry and neurological sciences. 1990;239(6):370-2.

3. Ihori N, Araki S, Ishihara K, Kawamura M. A case of frontotemporal lobar degeneration with progressive dysarthria. Behavioural neurology. 2006;17(2):97-104.

4. Lashley T, Rohrer JD, Mead S, Revesz T. Review: an update on clinical, genetic and pathological aspects of frontotemporal lobar degenerations. Neuropathology and applied neurobiology. 2015;41(7):858-81.

5. Lee EB, Porta S, Michael Baer G, Xu Y, Suh E, Kwong LK, et al. Expansion of the classification of FTLD-TDP: distinct pathology associated with rapidly progressive frontotemporal degeneration. Acta neuropathologica. 2017;134(1):65-78.

6. Montine TJ, Phelps CH, Beach TG, Bigio EH, Cairns NJ, Dickson DW, et al. National Institute on Aging-Alzheimer's Association guidelines for the neuropathologic assessment of Alzheimer's disease: a practical approach. Acta neuropathologica. 2012;123(1):1-11.

7. Broussolle E, Bakchine S, Tommasi M, Laurent B, Bazin B, Cinotti L, et al. Slowly progressive anarthria with late anterior opercular syndrome: a variant form of frontal cortical atrophy syndromes. Journal of the neurological sciences. 1996;144(1-2):44-58.

8. Otsuki M, Nakagawa Y, Mori F, Tobioka H, Yoshida H, Tatezawa Y, et al. Progressive anterior operculum syndrome due to FTLD-TDP: a clinico-pathological investigation. Journal of neurology. 2010;257(7):1148-53.

9. Deramecourt V, Lebert F, Maurage CA, Fernandez-Gomez FJ, Dujardin S, Colin M, et al. Clinical, neuropathological, and biochemical characterization of the novel tau mutation P332S. Journal of Alzheimer's disease : JAD. 2012;31(4):741-9.

10. Rektorova I, Matej R. Anterior opercular syndrome in frontotemporal lobar degeneration with ubiquitin-only immunoreactive neuronal changes. European journal of neurology. 2007;14(6):697-700. 